\title{
Dynamic characteristics and VIV of deepwater riser with axially varying structural properties
}

\author{
Weimin Chen ${ }^{\mathrm{a}, *}$, Min Li ${ }^{\mathrm{b}}$, Zhongqin Zheng ${ }^{\mathrm{a}}$, Tiancai Tan ${ }^{\mathrm{b}}$ \\ ${ }^{a}$ Key Laboratory of Environmental Mechanics, Institute of Mechanics, Chinese Academy of Sciences, Beijing 100190, China \\ ${ }^{\mathrm{b}}$ School of Aeronautics Sciences and Engineering, Beijing University of Aeronautics and Astronautics, Beijing 100191, China
}

\section{A R T I C L E I N F O}

\section{Article history:}

Received 15 July 2011

Accepted 27 December 2011

Editor-in-Chief: A.I. Incecik

Available online 25 January 2012

Keywords:

Deepwater riser

Varying tension

Vortex-induced vibration

Dynamic characteristics

\begin{abstract}
A B S T R A C T
A time-domain approach for predicting dynamic characteristics and vortex-induced vibration (VIV) response of deepwater risers is proposed based on finite element simulation combined with a hydrodynamic model. Effects of axially varying structural parameters, i.e. the effective tension and bending stiffness, on the dynamic characteristics and VIV of slender riser are examined using the presented approach. Our numerical results indicate that axially varying structural parameters can efficiently change the modal wave length as well as the modal displacement. The vibration amplitude of riser VIV response is influenced by the complex effect of factors involving the axial tension, bending stiffness and modal wave length. Generally speaking, for lower modes the response amplitude is larger at the axial position where the tension is smaller, whereas for higher modes the response amplitude is larger at the axial position where the bending stiffness is lower. Moreover, vibration wave velocity increases with increasing modal wave length along the riser length.
\end{abstract}

(c) 2011 Elsevier Ltd. All rights reserved.

\section{Introduction}

As the oil exploitation extends to deepwater ocean, the length of riser gets larger and the structural configuration becomes more complex, e.g. the outer or inner diameter gradually varies along the length of riser rather than a constant value. Additionally, the axial tension is no longer regarded as a constant along the overall length of riser due to considerable structure weight. Therefore, the dynamic analysis, such as the dynamic characteristics and VIV, of slender riser with varying structural properties has been facing new challenges (Grant et al., 2000; Liao and Vandiver, 2002; Sarpkaya, 2004).

In this study, first, a coupling analysis approach in timedomain for dynamic characteristics and VIV of deepwater riser, by means of finite element simulation combined with a hydrodynamic model taking account of the interaction between fluid and structure, is developed. Generally, the hydrodynamic force such as vortex-induced lift depends on the ambient fluid dynamics and structure motion; therefore, it is usually described by a function of the variables related to not only fluid but also structure. However, when such model is used to calculate the dynamic response, an iterative calculation is needed (Bokaian, 1994; Chen et al., 2009; Gei et al., 2007; Lyons and Patel, 1986)

\footnotetext{
* Corresponding author. Tel.: +86 1082543891 ; fax: +86 1082338527 .

E-mail address: wmchen@imech.ac.cn (W. Chen).
}

because the vibration amplitude of structure is unknown before the calculation. Here, an alternative lift model expressed by the instantaneous motion, rather than the final response amplitude, of structure is proposed so as to avoid costly iterations, and be more efficient for time domain calculation using finite element code. Based on the presented approach, the dynamic characteristics and VIV of the riser with axially varying structural properties are examined. It is observed that the distributions of the modal wave length and displacement are significantly influenced by the axial variation of structural properties. The VIV amplitude depends on the overall effect of multiple factors involving tension, stiffness and modal wave length.

\section{Numerical modeling}

\subsection{Hydrodynamic model and finite element simulation}

The dynamic equilibrium equation of a tension Euler beam (Sarpkaya, 2004) is as follows:

$m \frac{\partial^{2} y(x, t)}{\partial t^{2}}+c \frac{\partial y(x, t)}{\partial t}+\frac{\partial^{2}}{\partial x^{2}}\left[E I(x) \frac{\partial^{2} y(x, t)}{\partial x^{2}}\right]-\frac{\partial}{\partial x}\left[T(x) \frac{\partial y(x, t)}{\partial x}\right]=f(x, t)$

where $y(x, t)$ is the displacement vertical to the beam axis, $x$ is the axial position and $t$ is the time; $m$ and $c$ are the structural mass and damping per unit length, respectively, $\operatorname{EI}(x)$ and $T(x)$ are the 
bending stiffness and axis tension, respectively. $f(x, t)$ is the hydrodynamic force consisting of the vortex-induced lift force $f_{v}(x, t)$ and motion-induced fluid damping force $f_{f}(x, t)$, which are given respectively as follows:

$f_{v}(x, t)=(1 / 2) C_{L} \rho V^{2} D \sin \left(\omega_{v} t\right)$

$f_{f}(z, t)=\frac{1}{2} C_{D} \rho D(V-\dot{y})|V-\dot{y}|+C_{A} \frac{1}{4} \pi D^{2} \rho(\dot{V}-\ddot{y})+\frac{1}{4} \pi D^{2} \rho \dot{V}$

where $C_{L}$ is the vortex-induced lift coefficient, $\rho$ and $V$ are the density and velocity of fluid, respectively, $D$ is the diameter of riser and $\omega_{v}$ is the vortex shedding frequency. $C_{D}$ and $C_{A}$ are the drag and added mass coefficient, respectively, of which values can be determined by experiments.

Generally, $C_{L}$ is expressed as a function of the variables indicating structural motion so as to take account of the interaction between fluid and structural dynamic. Thus existing lift model is presented as a hyperbolical curve of $C_{L}$ against structural motion amplitude (see Fig. 1), of which the coefficients such as $C_{L \text { max }}$ and $C_{L A O}$ are based on experimental data. However, before the calculation the motion amplitude is not known. Thus, an iterative calculation is used in the existing model, which is computationally expensive and unfortunately is unsuitable to the time domain calculation of dynamic response simulated using finite element method (FEM) codes. Therefore, here an alternative lift curve expressed by the instantaneous motion of structure is proposed.

The proposed lift coefficient $C_{L}$, based on which the lift force (Eq. (2a)) inputs the same energy to the structure in a motion period as the existing one does, is assumed to be a function of structural velocity in the form of

$C_{L}(\dot{y})=C_{L 0}+a \dot{y}+b \dot{y}^{2}+c \dot{y}^{3}$

where the coefficients $C_{L o}, a, b$ and $c$ will be determined as follows. The structural motion in lock-in is assumed as $y=A \sin (\omega t)$. Then the energies input, respectively by the lift force $F=F_{0} \cos (\omega t)$ with a constant amplitude $F_{0}=(1 / 2) 2 C_{L O} \rho V^{2} D$ and the unsteady lift force $F_{1}$ in the form of

$F_{1}=F_{0} \cos (\omega t)+a^{\prime} \dot{y}+b^{\prime} \dot{y}^{2}+c^{\prime} \dot{y}^{3}$

in a one-fourth period can be written as

$W_{0}=\int_{0}^{T / 4} F_{0} A \omega(\cos \omega t)^{2} d t=\frac{\pi}{4} F_{0} A$

$W_{1}=\int_{0}^{T / 4} F_{1} A \omega \cos \omega t d t=\frac{\pi}{4} F_{0} A+a^{\prime} A^{2}+b^{\prime} A^{3}+c^{\prime} A^{4}$

or

$W_{1} / W_{0}=1+a^{\prime \prime} A+b^{\prime \prime} A^{2}+c^{\prime \prime} A^{3}$

where $a^{\prime \prime}=\left(\omega / F_{0}\right) a, b^{\prime \prime}=\left(8 \omega^{2} / 3 \pi F_{0}\right) b$ and $c^{\prime \prime}=\left(3 \omega^{3} / 4 F_{0}\right) c$. Therefore, a new lift coefficient $C_{L}^{\prime \prime}$ can be written as

$C_{L}^{\prime \prime}=C_{L 0}\left(1+a^{\prime \prime} A+b^{\prime \prime} A^{2}+c^{\prime \prime} A^{3}\right)$

The values of $a, b, c$ and $C_{L 0}$ can be resolved by fitting the new curve with the original one at some key points, e.g. points B1, B2

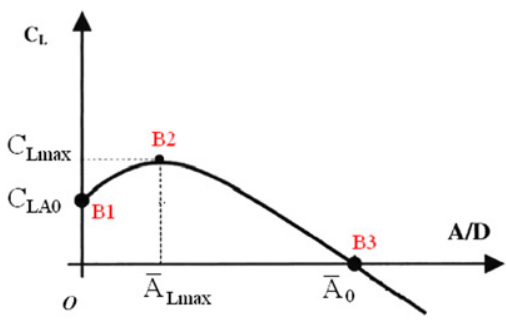

Fig. 1. Lift curve. and B3 in Fig. 1. A group of equations describing the relationship between the previous curve and the new curve is as follows:

$C_{L 0}=C_{L A 0}$

$C_{L 0}\left[1+\frac{\omega}{F_{0}} a \bar{A}_{L \max }+\frac{8 \omega^{2}}{3 \pi F_{0}} b\left(\bar{A}_{L \max }\right)^{2}+\frac{3 \omega^{3}}{4 F_{0}} c\left(\bar{A}_{L \max }\right)^{3}\right]=C_{L \max }$

$\frac{\omega}{F_{0}} a+\frac{16 \omega^{2}}{3 \pi F_{0}} b \bar{A}_{L \max }+\frac{9 \omega^{3}}{4 F_{0}} c\left(\bar{A}_{L \max }\right)^{2}=0$

$C_{L}^{\prime \prime}\left[1+\frac{\omega}{F_{0}} a \bar{A}_{0}+\frac{8 \omega^{2}}{3 \pi F_{0}} b\left(\bar{A}_{0}\right)^{2}+\frac{3 \omega^{3}}{4 F_{0}} c\left(\bar{A}_{0}\right)^{3}\right]=0$
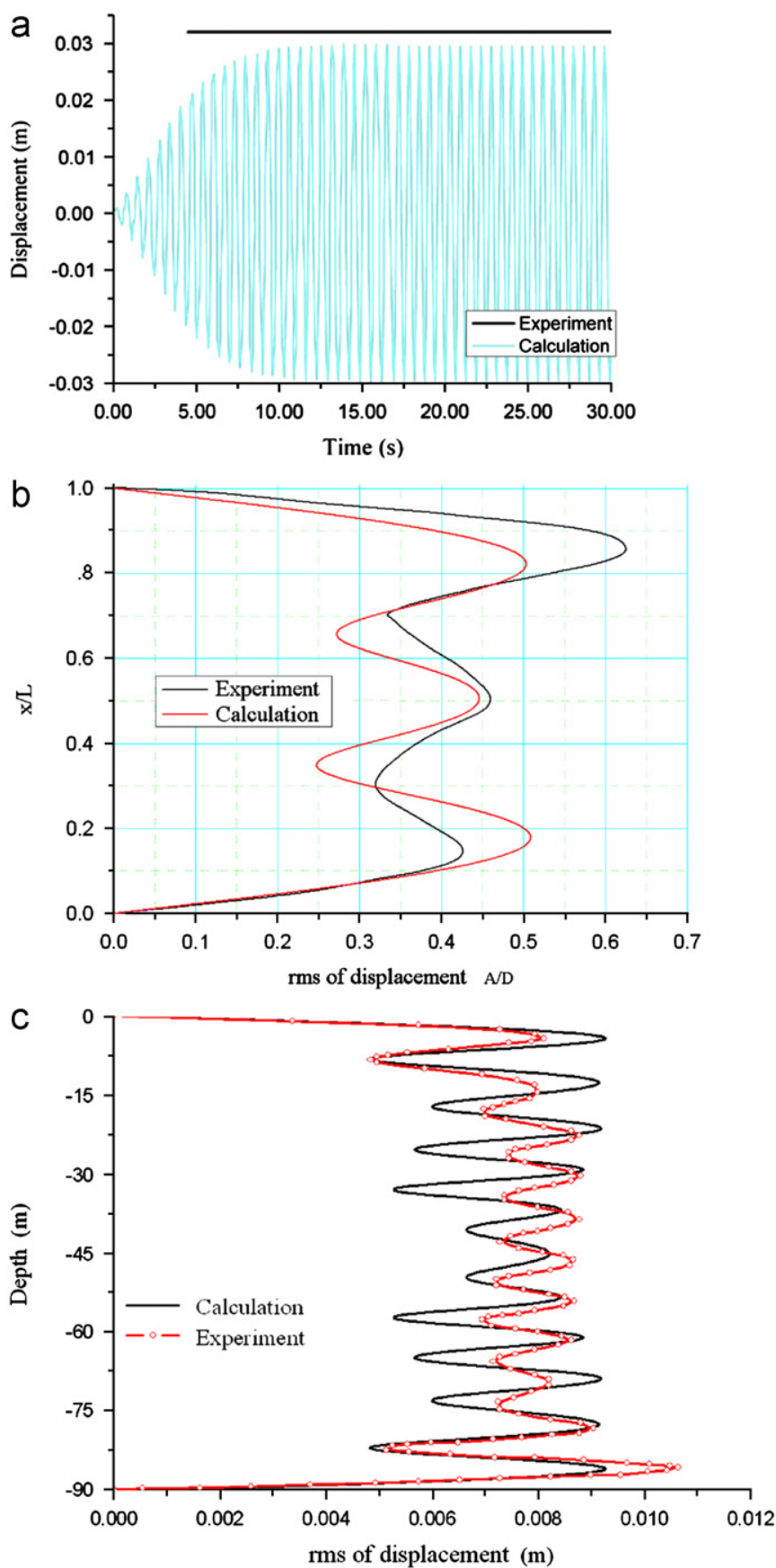

Fig. 2. Comparisons of VIV response between the presented numerical simulation and the existing experimental results of (a) rigid cylinder in uniform flow, (b) flexible cylinder in stepped flow and (c) flexible cylinder in sheared flow. 
where $\bar{A}_{0}$ and $\bar{A}_{L \max }$ is the amplitudes when $C_{L}=0$ and $C_{L}=C_{L \max }$, respectively (as shown in Fig. 1).

Based on the presented lift model, finite element simulation can be carried out for the dynamic response of a riser undergoing VIV.

\subsection{Validation against experimental results}

To validate the proposed model, the numerical results are compared to experimental results (see Fig. 2), i.e. a rigid cylinder undergoing uniform flow by Khalak and Williamson (1999), and the flexible cylinders, respectively undergoing a stepped flow by Chaplin et al. (2005) and a sheared flow by Trim et al. (2005).

In the numerical simulations, the hydrodynamic coefficients are set to be $C_{A}=1.0, C_{d}=1.2$, which were determined based on the corresponding experiments (Sarpkaya, 2004; Lyons and Patel, 1986,). Considering that the lift force for a rigid cylinder is generally higher than a flexible cylinder, we set the lift coefficients to be $C_{L}=0.5+1.82 A-1.29 A^{2}-0.707 A^{3}$ and $C_{L}=0.22+$ $1.62 A-2.31 A^{2}+0.754 A^{3}$ for rigid and flexible cylinder, respectively (Liao and Vandiver, 2002; Zhang and Chen, 2010). Fig. 2 indicates that the calculated amplitudes agree with experimental results for both rigid and flexible cylinders in various flow fields.

\section{Numerical results and discussions}

In practice, especially for deepwater riser, the structural configuration is usually complex, e.g. the outer or inner diameter may gradually vary along the riser length rather than keeping a constant value and the axial tension is no longer regarded as a constant along the overall length of riser due to the considerable structure weight. Therefore the structural property along the overall length of riser is not uniform yet. In this section, the dynamic characteristics and VIV of the riser with axially varying structural properties, i.e. axial tension and bending stiffness, are examined.

\subsection{Effect of axially varying tension on dynamic characteristics}

The riser (shown in Fig. 3) is $1000 \mathrm{~m}$ long and the outer and inner diameters are $0.500 \mathrm{~m}$ and $0.445 \mathrm{~m}$, respectively. The top tension $T$ is $6.24 \times 10^{6} \mathrm{~N}$ (at the right end of the riser) and the top tension factor $T / W$ is 1.57 ( $W$ is the riser weight). Because of the effect of the riser weight $W$ (along the negative direction of the $x$-axis), the tension along the overall riser length decreases linearly from the right end to the left end of the riser, or the riser has an axially varying tension but not a constant tension.

The natural frequencies of the riser with axially varying tension are shown and compared with that of the riser with a constant tension (as the effect of structural weight is neglected) in Fig. 4. The selected mode shapes are presented in Fig. 5. Fig. 4 indicates that the frequency of the riser with linearly decreasing tension along the riser length gets smaller than the frequency of the riser with a constant tension. Observing the modal shapes in Fig. 5, we can see that the wave length of the modal shape axially varies with the variation of riser tension, or it becomes longer in regions of higher tension along the riser length; also, the amplitude of each peak axially varies with the riser tension, it is

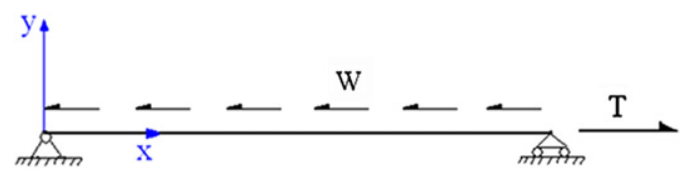

Fig. 3. Sketch of the riser with varying tension.

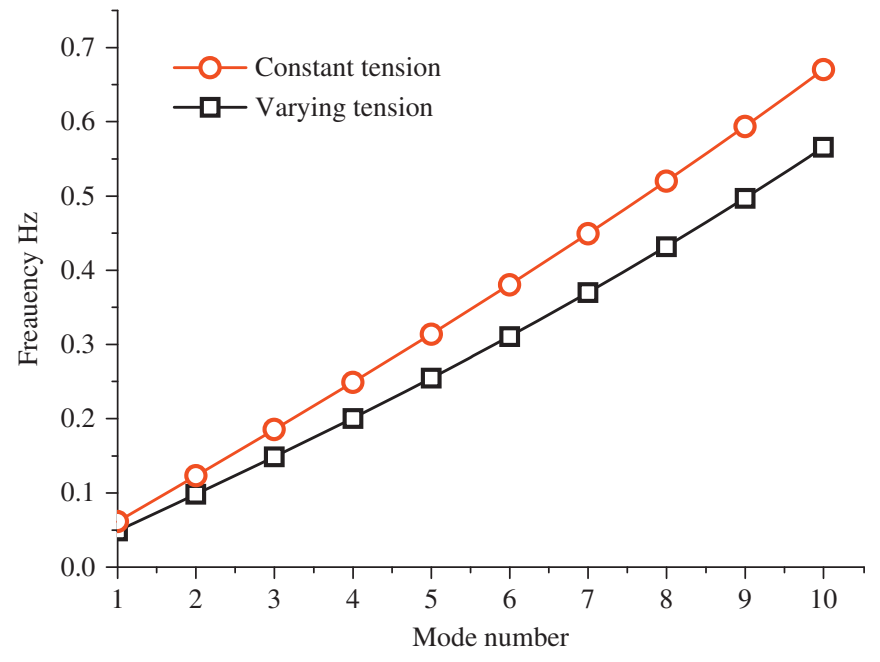

Fig. 4. Natural frequencies of riser with varying and constant tensions.

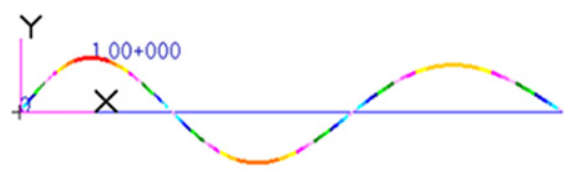

Mode 3

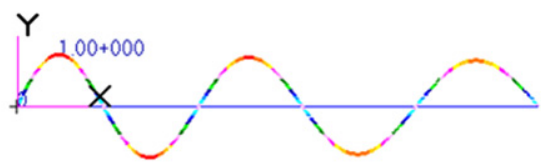

Mode 5

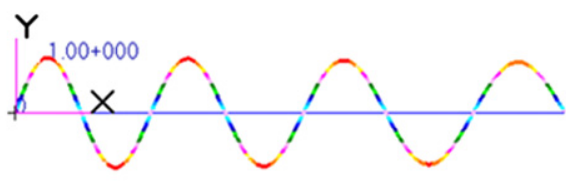

Mode 7
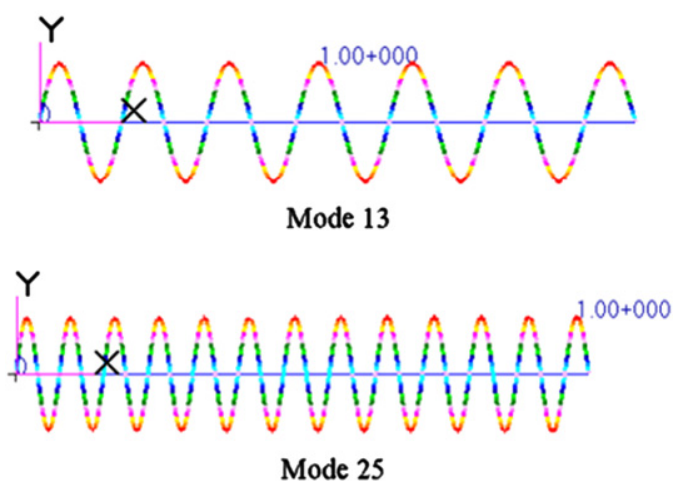

Fig. 5. Modal shape of riser with varying tension dropping from right to left.

interesting that the maximum peak of the amplitude moves from regions of lower tension to regions of higher tension as the mode number increases. The theoretical explanations about these phenomena will be discussed later.

\subsection{Effect of axially varying stiffness on dynamic characteristics}

In practice, the wall thickness of some deepwater risers often typically changes along the riser length for reason of material and structural efficiencies. Here, the variation of bending stiffness 
$E I(x)$ is considered by varying the wall thickness. The riser is divided into five parts with the same outer diameter but different inner diameters, i.e. $0.365 \mathrm{~m}, 0.385 \mathrm{~m}, 0.405 \mathrm{~m}, 0.425 \mathrm{~m}$ and $0.445 \mathrm{~m}$, where the stiffness is higher at the bottom of the riser. The gravity is neglected here, or the tension has a constant value, $6.24 \times 10^{6} \mathrm{~N}$, along overall riser length.

Selected mode shapes are presented in Fig. 6. It shows that because of the axial decrease in stiffness, both wave length and amplitude axially increase. The distribution of the amplitude along the riser length is approximately inversely proportional to the distribution of the stiffness $\operatorname{EI}(x)$ that becomes more evident for higher mode, e.g. mode 43 (the last plot in Fig. 6).

\subsection{VIV of riser with axially varying structural properties}

The parameters of the riser are same with those in Section 3.2. Additionally, the gravity is taken into account. Two cases of top tensions $T=1.91 \times 10^{7} \mathrm{~N}$ and $T=6.24 \times 10^{6} \mathrm{~N}$ are examined. As we know, the motion of top end of ocean platform induced by the environmental wave and current may introduce pronounced influence on submarine structure (Huse et al., 1999; Lie and Kaasen, 2006; Chen et al., 2006). For example, the vertical motion (the heave) of the platform may make the riser tension change remarkably, or even, in extreme case, into negative value at certain positions along the riser length.

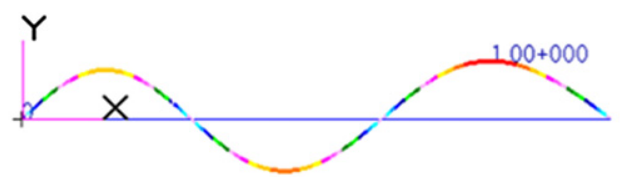

Mode 3

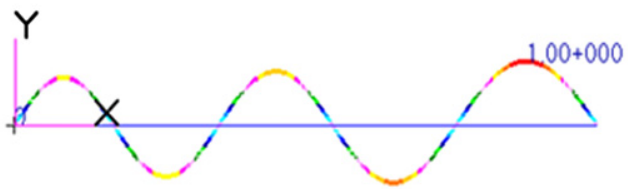

Mode 5
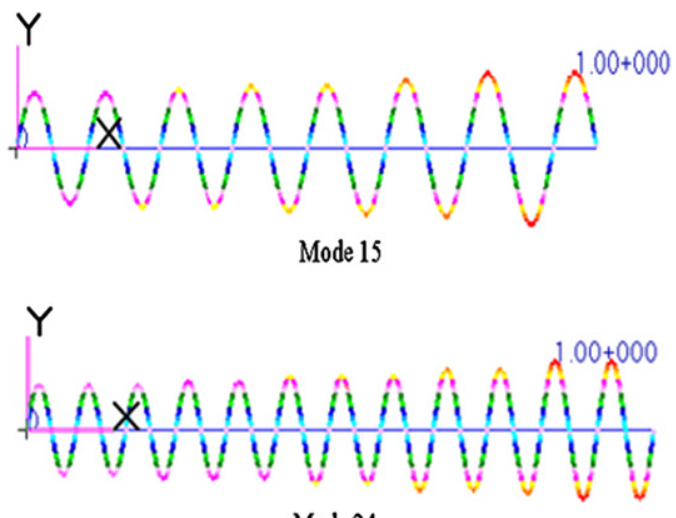

Mode 24

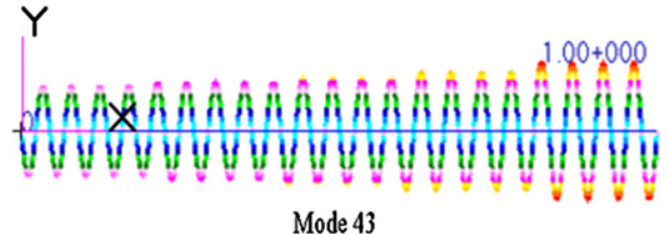

Fig. 6. Modal shape of riser with varying stiffness increasing from right to left.
In our FEM simulations, the hydrodynamic forces involving the vortex-induced lift $f_{v}(x, t)$ and the fluid damping force $f_{f}(x, t)$ are exerted on the riser. The vortex-induced lift force $f_{v}(x, t)$ is exerted at the centers of modal shape, and the fluid damping force $f_{f}(x, t)$ is uniformly distributed along the riser. The frequencies of the lift force are consistent with the natural frequencies of the riser modes ranging from mode 1 to 35 . Then, the VIV responses are calculated numerically by the FEM model presented above.

For case of higher tension, $T=1.91 \times 10^{7} \mathrm{~N}$, selected results, the modal shape and VIV displacement of mode 27 , are presented in Fig. 7 where the vortex-induced lift force is loaded at the seventh trough from the left end of the riser, i.e. $x / L=0.47$. The mode shape is shown in Fig. 7a, as well the temporal-spatial
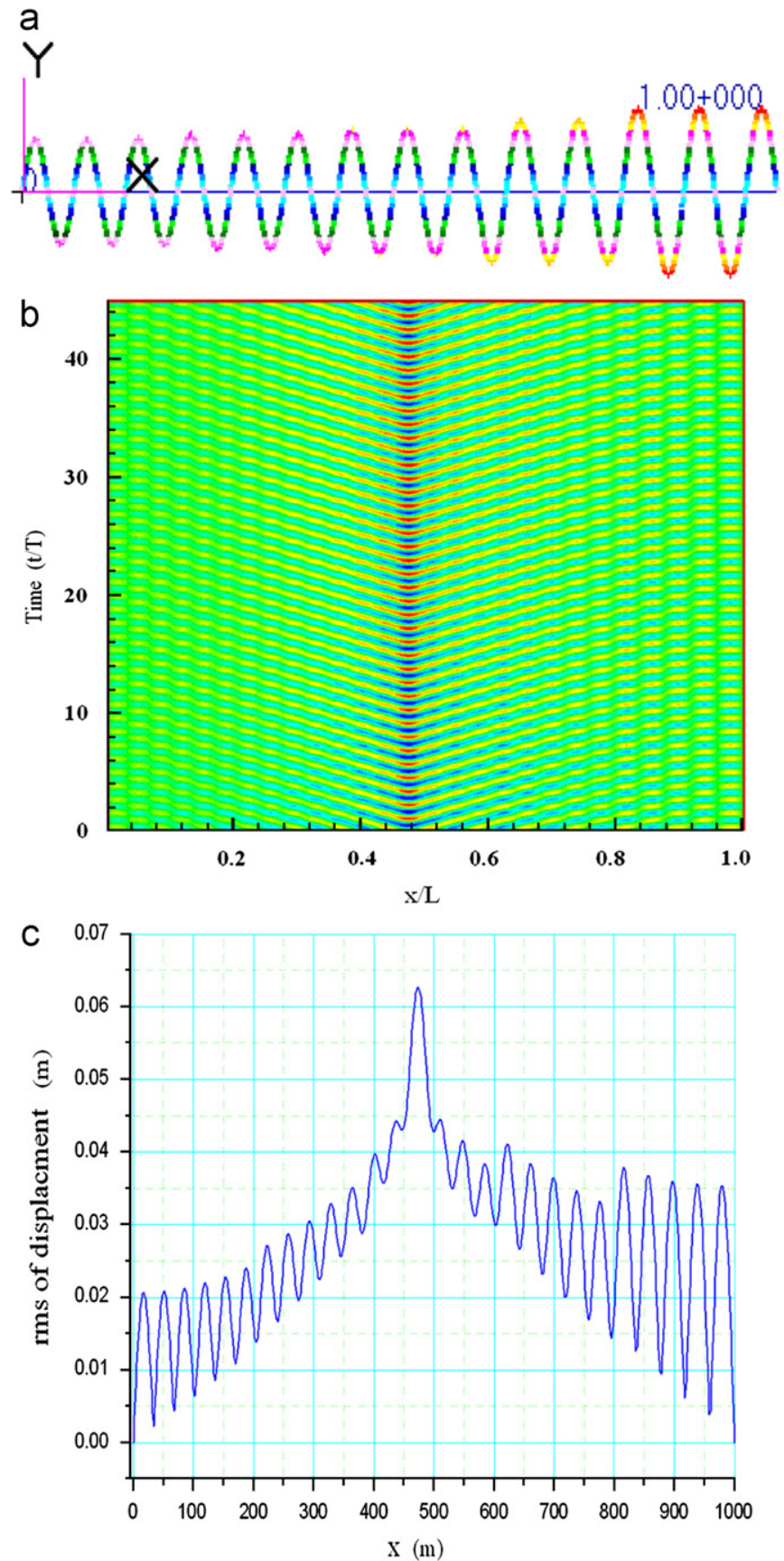

Fig. 7. Mode shape and VIV response of riser with axially varying structura properties (mode 27, tension $T=1.91 \times 10^{7} \mathrm{~N}$ ): (a) mode shape of mode 27, (b) temporal-spatial evolution of VIV displacement and (c) rms of displacement. 
evolution and rms of displacement are plotted in Fig. 7b and c, respectively.

The results of displacement response (shown in Fig.7b and c) indicate that the vibration of the riser is dominated by an intermediate wave (Zhang and Chen, 2010; Vandiver, 1993) between standing and travelling wave. The displacement attenuates slowly at the right part of the riser and quickly at the left part due to the increase of structural bending stiffness from the right to left of the riser, or the displacement at the right part of the riser is larger than the displacement at the left part, this amplitude distribution is similar with that of the mode shape. The explanation of above phenomenon might be that the amplitude of displacement depends not only on the attenuation velocity, but also the propagation distance because of the influence of

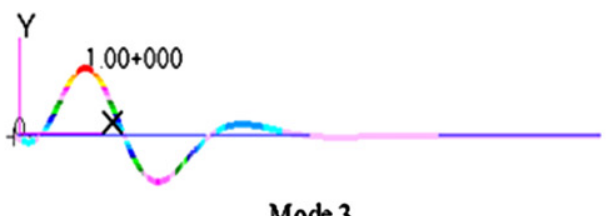

\section{Mode 3}
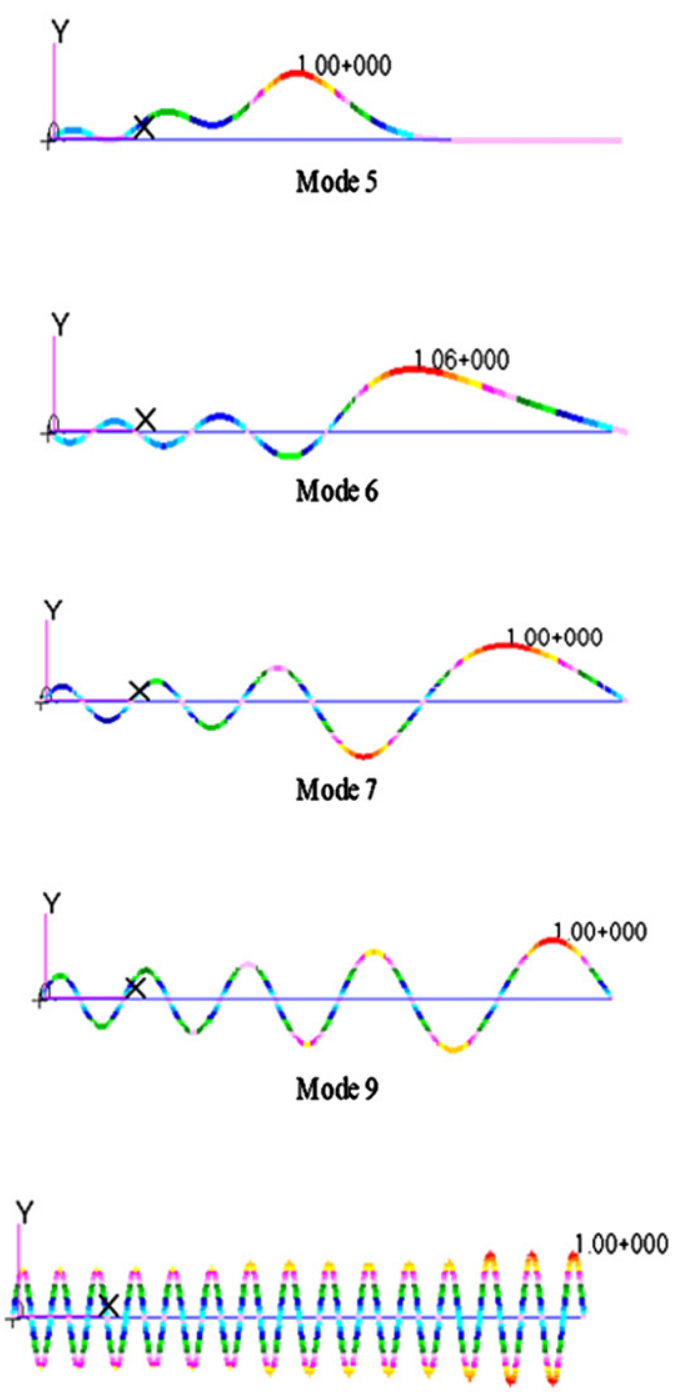

Mode 29

Fig. 8. Selected modal shapes of riser with varying stiffness for case of lower tension $T=6.24 \times 10^{6} \mathrm{~N}$. travelling wave. Additionally, Fig. $7 \mathrm{~b}$ and $\mathrm{c}$ show that the wave velocity becomes faster as the wave length increases.

For case of lower tension, $T=6.24 \times 10^{6} \mathrm{~N}$, less than structural weight, the mode shapes for selected modes, i.e. modes $3,5,6,7$, 9, 11 and 29, are presented in Fig. 8. It shows that a sinusoidal approximation is only appropriate for higher modes (higher than mode 7). For lower modes there is almost no displacement in the regions of higher tension.

Among the calculated VIV results, the abrupt displacement augmentation at the position with negative tension is observed, as shown in Fig. 9. The maximum augmentation is around $10-20$ times, which may introduce instability and even consequent damage of structure, thus this unfavorable case should be noted.

\subsection{Discussion}

For a tension beam written as Eq. (1), if the displacement is assumed as $y=\bar{y}(x) e^{-i \omega t}$ and the right-hand side of Eq. (1) is set to be zero, the governing equation can be rewritten as

$E I(x) \frac{d^{4} \bar{y}}{d x^{4}}+\frac{d(E I)}{d x} \frac{d^{3} \bar{y}}{d x^{3}}+\frac{d^{2}(E I)}{d x^{2}} \frac{d^{2} \bar{y}}{d x^{2}}-T \frac{d^{2} \bar{y}}{d x^{2}}-\frac{d T}{d x} \frac{d \bar{y}}{d x}-m(x) \omega^{2} \bar{y}-i c(x) \omega \bar{y}=0$

By virtue of the WKB method (Liao and Vandiver, 2002), we let

$\bar{y}(x)=e^{i \theta(x)}\left\{a_{0}(x)+a_{1}(x)+a_{2}(x)\right\}$

where $a_{1}$ and $a_{2}$ are higher order corrections. Also, we let

$d \theta(x) / d x=\gamma(x)$

where $\gamma(x)$ is the local wave number. Substituting Eqs. (10) and (11) into Eq. (9) and remaining the leading order terms yields the dispersion relation as follows:

$E I(x) \gamma^{4}(x)+T(x) \gamma^{2}(x)-m(x) \omega^{2}-i c(x) \omega=0$

Remaining the next order terms yields

$E I\left(-6 i \gamma^{\prime} \gamma^{2} a_{0}+\gamma^{4} a_{1}-4 i \gamma^{3} a_{0}^{\prime}\right)+2(E I)^{\prime}\left(-i \gamma^{3} a_{0}\right)-T\left(i \gamma^{\prime} a_{0}-\gamma^{2} a_{1}+2 i \gamma a_{0}^{\prime}\right)$

$$
-T^{\prime}\left(i \gamma a_{0}\right)-m \omega^{2} a_{1}-i c \omega a_{1}=0
$$

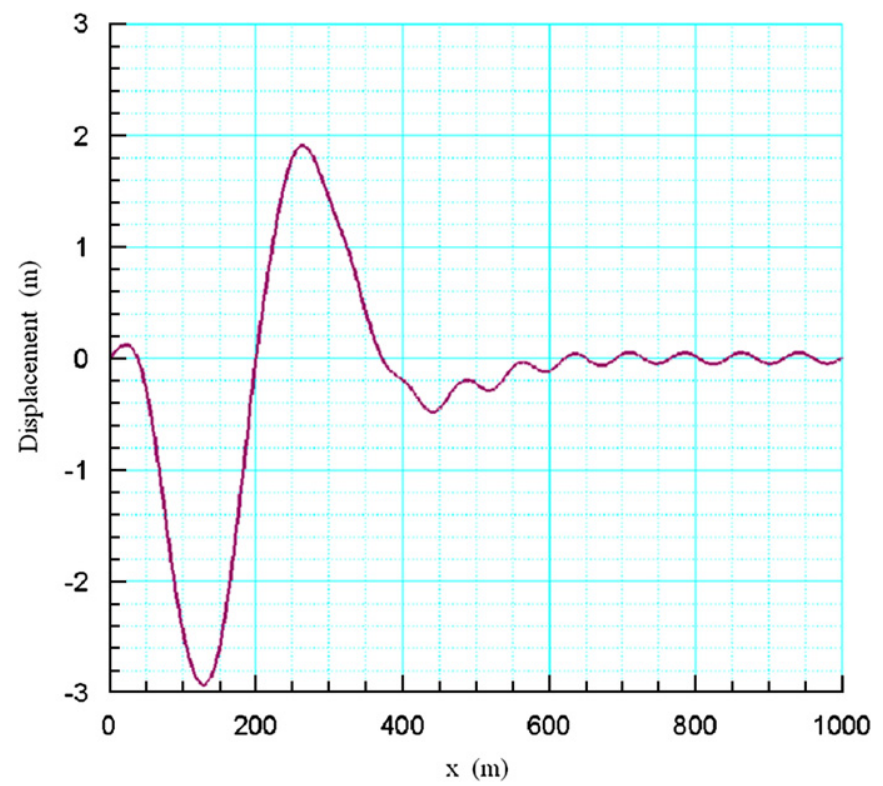

Fig. 9. VIV response of riser with varying stiffness for case of lower tension $T=6.24 \times 10^{6} \mathrm{~N}$ 
Eliminating all terms with $a_{1}$ by means of Eq. (12) yields that the amplitude $a_{0}(x)$ along the riser axis can be written as

$a_{0}^{2}(x)\left[E I(x) \gamma^{3}(x)+(1 / 2) T(x) \gamma(x)\right]=$ cons.

According to Eq. (14), for the case of beam with axially varying tension, wave number $\gamma(x)$ would become smaller or wave length $\lambda(x) \quad(\lambda(x)=(2 \pi / \gamma(x)))$ would become larger with increasing tension $T(x)$. That is consistent with the modal shapes shown in Fig. 5. The value of amplitude $a_{0}(x)$ depends on the value of the terms in the square bracket of Eq. (14). For modal shape of lower mode, the second term in the square bracket, $(1 / 2) T(x) \gamma(x)$, dominates the total value of the square bracket due to a stronger influence of the tension than the bending stiffness. So at position $x$ with larger tension $T(x)$, the value of the square bracket is larger, and then the amplitude $a_{0}(x)$ would be smaller because the product value of the two terms is constant as written in Eq. (14). However, for higher modes, the first term in the square bracket, i.e. $E I(x) \gamma^{3}(x)$, would mainly dominate the total value due to a stronger influence of the bending stiffness. So at position $x$ with larger tension $T(x)$ (or smaller $\gamma(x)$ ), the value of the square bracket is smaller because of the drop of $\operatorname{EI}(x) \gamma^{3}(x)$ value. That is to say, the amplitude $a_{0}(x)$ would be larger as shown in Eq. (14). The movement of the amplitude peak with increasing mode number can be seen in Fig. 5 .

Similarly, for the case of a beam with axially varying bending stiffness $E I(x)$, amplitude peak would move along the beam length from the position with larger stiffness $\operatorname{EI}(x)$ to the position with smaller $E I(x)$ as the mode number increases (see Fig. 6).

\section{Conclusions}

An approach, for predicting dynamic characteristics and VIV of deepwater riser, by finite element simulation combined with a coupling hydrodynamic model in time domain is proposed. Compared with the experiments, satisfied agreement of displacement response is observed. Effects of axially varying structural properties are examined. Based on our numerical results, we draw the following conclusions:

1) Along the riser length, modal wave length increases with increasing tension or decreasing bending stiffness.

2) Modal amplitude is influenced by axially varying tension, stiffness and wave number (or wave length) as described in Eq. (14). Generally, for lower modes the amplitude gets larger at the axis position where tension is smaller, whereas for higher modes the amplitude gets larger at the axis position where bending stiffness is lower.

3) For the case of VIV response, vibration wave velocity would increase, i.e. the vibration would propagate faster along the riser, due to the increasing modal wave length.
The displacement augmentation, around 10-20 times, at the position with lower tension may introduce instability and consequent damage of the riser structure that should be paid attention in practical engineering. It is also noted that the presented approach cannot fully capture the physics of VIV such as the wake-jumping and hysteresis since it is virtually based on a semi-empirical lift curve.

\section{Acknowledgments}

This work is supported by the Intellectual Innovation Project of the Chinese Academy of Sciences (Grant no. KJCX2-YW-L07) and the Fundamental Research Funds for the Central Universities (Grant no. YWF-10-01-B05).

\section{References}

Bokaian, A., 1994. Lock-in prediction of marine risers and tethers. J. Sound Vib. 175 (5), 607-623.

Chaplin, J.R., Bearman, P.W., Huera, F.J., et al., 2005. Laboratory measurements of vortex-induced vibrations of a vertical tension riser in a stepped current. J. Fluid Struct. 21, 3-24.

Chen, W.M., Zhang, L.W., Li, M., 2009. Prediction of vortex-induced vibration of flexible riser using an improved wake-oscillator model. In: Proceedings of the ASME 28th International Conference on Ocean, Offshore and Arctic Engineering OMAE 2009, May 2009, Honolulu, Hawaii.

Chen, X.H., Ding, Y., Zhang, J., et al., 2006. Coupled dynamic analysis of a mini TLP: comparison with measurements. Ocean Eng. 33, 93-117.

Gei, F., Hui, L., Hong, Y.S., 2007. Vortex-induced vibration of submarine floating tunnel undergoing shear flow. J. Grad. Sch. Chin. Acad. Sci. 24 (3), 352-356. (in Chinese).

Grant, R., Litton, R., Finn, L., Maher, J., Lambrakos, K., 2000. Highly compliant rigid risers: Field test benchmarking a time domain VIV algorithm. In: Proceedings of the Offshore Technology Conference, Houston, Texas, Offshore Technology Conference, OTC 11995.

Huse, E., Kleiven, G., Nielsen, F.G., 1999. VIV-induced axial vibration on deep sea risers. In: Proceedings of the Offshore Technology Conference, Houston, Texas, Offshore Technology Conference, OTC 10932.

Khalak, A., Williamson, C.H.K., 1999. Motions, forces and mode transitions in vortex-induced vibrations at low mass-damping. J. Fluid Struct. 13, 813-851.

Liao, J.C., Vandiver, J.M., 2002. Vortex-Induced Vibration of Slender Structure in Unsteady Flow. Ph.D. Dissertation, Massachusetts Institute of Technology.

Lie, H., Kaasen, H.K., 2006. Modal analysis of measurements from a large-scale VIV model test of a riser in linearly sheared flow. J. Fluid Struct. 22, 557-575.

Lyons, G.J., Patel, M.H., 1986. A prediction technique for vortex induced transverse response of marine risers and tethers. J. Sound Vib. 111 (3), 467-487.

Sarpkaya, T., 2004. A critical review of the intrinsic nature of vortex-induced vibration. J. Fluids Struct. Mech. 46, 389-447.

Trim, A.D., Braaten, H., Lie, H., et al., 2005. Experimental investigation of vortexinduced vibration of long marine risers. J. Fluid Struct. 21, 335-361.

Vandiver, J.K., 1993. Dimensionless parameters important to the prediction of vortex-induced vibration of long, flexible cylinders in ocean currents. J. Fluid Struct. 7 (5), 423-455.

Zhang, L.W., Chen, W.M., 2010. Study on the parameters for determining response types of long flexible riser undergoing VIV in deepwater. China Offshore Oil and Gas 22 (3), 202-206. (in Chinese). 